\title{
Neuroprotective effects of curdione against focal cerebral ischemia reperfusion injury in rats
}

\author{
This article was published in the following Dove Press journal: \\ Neuropsychiatric Disease and Treatment \\ 30 June 2017 \\ Number of times this article has been viewed
}

\section{Xing-Jie Li ${ }^{1}, *$ \\ Li Liang ${ }^{2, *}$ \\ Hong-Xia Shi' \\ Xiao-Ping Sun' \\ jing Wang' \\ Lian-Sheng Zhang'}

'Health Management Center, Lanzhou University Second Hospital, Lanzhou, People's Republic of China; ${ }^{2}$ Department of Pharmacy, Lanzhou University Second Hospital, Lanzhou, People's Republic of China

*These authors contributed equally to this work
Correspondence: Lian-Sheng Zhang Health Management Center, Lanzhou University Second Hospital, Cuiying Men Road 82, Lanzhou 730030, People's Republic of China

Tel/fax +86 93I 8942930

Email lzulkgl@।63.com
Background: Curdione is one of the most highly concentrated component of the active constituents in E-zhu, which has been reported to possess a variety of activities. However, the pharmacologic neuroprotective activity of curdione has not been evaluated. The present study aimed to investigate the protective effect of curdione on focal cerebral ischemia reperfusioninduced injury in rats and further exploring the underlying mechanisms.

Materials and methods: Adult male Sprague Dawley rats were subjected to middle cerebral artery occlusion (MCAO) surgery for $2 \mathrm{~h}$, followed by reperfusion stage. All animals received treatment once a day for 7 days before surgery and 14 days from $4 \mathrm{~h}$ after the reperfusion started. The neurological deficit test and Morris water maze test were performed at 1, 4, 7 and 14 days after MCAO. The infarct size of animals was determined by the 2,3,5-triphenyltetrazolium chloride staining, and pathological brain damage was estimated by hematoxylin-eosin staining. The malonaldehyde (MDA) levels and the activities of superoxide dismutase (SOD), catalase (CAT) and glutathione peroxidase (GSH-PX) were detected by enzyme-linked immunosorbent assay. Expression of apoptotic proteins was measured by Western blot.

Results: Our results showed that curdione could significantly reduce the infarct size and neurological deficits, promote cognitive function recovery and recover neuronal morphologic damages in MCAO rats. It also blocked the increase of MDA content and elevated the activities of SOD, CAT and GSH-PX. Moreover, curdione attenuated the expression of Cyt-C, c-caspase-3 and c-caspase-9 increased the Bcl-2/Bax ratio and hence decreased the cellular apoptosis.

Conclusion: Curdione possessed potential neuroprotective effect on rats in the MCAO model. The anti-oxidative and anti-apoptotic properties may be involved in the underlying mechanisms.

Keywords: curdione, cerebral ischemia reperfusion, oxidative stress, apoptosis

\section{Introduction}

Ischemic stroke is one of the leading causes of human disability and mortality in the world, ${ }^{1,2}$ which has a profoundly negative impact on both the patients and the society. Thrombolysis with tissue plasminogen activator is the only efficient drug therapy for the treatment of ischemic stroke. ${ }^{3}$ However, the short time window for patients makes it unable to be applied wide bound, not to mention for long-term use. ${ }^{4}$ Therefore, it is very necessary to develop safe and effective compounds for stroke therapy. Multiple mechanisms are involved in the ischemic process that ultimately lead to neuron death. In particular, oxidative stress and neuron apoptosis after ischemia are the major pathways that drive neuronal cell death. ${ }^{5}$ Hence, exploring agents that target to both oxidative stress and apoptosis may be an effective therapy for ischemic diseases.

Curcuma zedoaria, the so-called E-zhu in Chinese, is a common folk medicine in China, which has been used to remove blood stasis and alleviate pain since ancient time. ${ }^{6}$ Many studies have demonstrated that the main bioactive components of E-zhu 
possess a variety of activities, such as antimicrobial, antiviral, anticancer, anti-inflammatory, anti-thrombotic and so on. ${ }^{7-10}$ Curdione is one of the most highly concentrated component of the active constituents, ${ }^{11}$ which was first isolated from C. zedoaria in 1966 by Hikino et al. ${ }^{12}$ Its chemical structure is shown in Figure 1. Dohare et $\mathrm{al}^{13}$ reported that curcuma oil has neuroprotective activity. However, the pharmacologic neuroprotective activity of curdione has not been evaluated to date.

Thus, the present study was aimed to investigate the potential therapeutic efficacy of curdione in rats with focal cerebral ischemia reperfusion injury. Moreover, further studies were carried out to clarify the possible underlying mechanisms.

\section{Materials and methods Animals}

Adult male Sprague Dawley rats weighing 240-270 g were purchased from Beijing Wei Tong Li Hua Experimental Technology Animal Co. Ltd. (Beijing, China). The research was conducted in accordance with the Declaration of Helsinki and the Guide for Care and Use of Laboratory Animals as adopted and promulgated by the United National Institutes of Health. All experimental protocols were approved by the Animal Care and Use Committee of Lanzhou University.

\section{Cerebral ischemia-reperfusion model}

The middle cerebral artery occlusion (MCAO) surgery was conducted as previously described. ${ }^{14}$ All rats were anesthetized with $10 \%$ chloral hydrate $(300 \mathrm{mg} / \mathrm{kg}$, intraperitoneally), and then, a intraluminal suture was inserted from the external carotid artery stump into the internal carotid artery of rats. After $2 \mathrm{~h}$, the suture was withdrawn and the blood circulation was recovered. The rats in the sham group underwent the same surgery without ligating the arteries. Animals were randomly assigned to three groups $(n=10)$ : 1) sham, 2) $\mathrm{MCAO}$, and 3) MCAO and curdione treatment $(100 \mathrm{mg} / \mathrm{kg}$, dissolved in 10\% Tween-80). Curdione was obtained from Pure-one Bio Technology, Co. Ltd. (Shanghai, China).

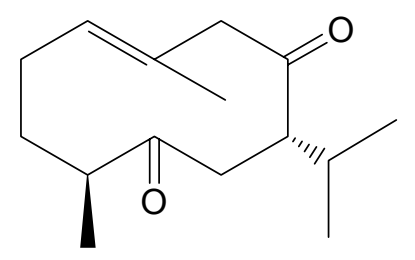

Figure I Chemical structure of curdione.
Vehicle or drugs were administered intragastrically once a day for 7 days before surgery and 14 days from $4 \mathrm{~h}$ after the start of reperfusion until the animals were sacrificed. In the sham and MCAO groups, a similar volume of $10 \%$ Tween- 80 solution was administrated.

\section{Neurological function assessment}

For all animals, behavioral tests were performed before MCAO and at 1, 4, 7 and 14 days after MCAO by an investigator who was blinded to the experimental groups. Neurological deficits were evaluated as previously reported, ${ }^{15}$ which included a set of modified neurological severity scores (NSSs) as shown in Table 1. NSS incorporates a series of motor, sensory, reflex and balance tests. ${ }^{16}$ In the tests, neurological function was graded on a scale of $0-18$; 1 point was awarded for the inability to perform the tasks or for the lack of a tested reflex, 13-18 points indicated severe

Table I Neurological severity scores

\begin{tabular}{|c|c|}
\hline Motor tests & Points \\
\hline \multicolumn{2}{|l|}{ Raising rat by the tail } \\
\hline Flexion of forelimb & 1 \\
\hline Flexion of hindlimb & 1 \\
\hline Head moved $>10^{\circ}$ to vertical axis within $30 \mathrm{~s}$ & 1 \\
\hline \multicolumn{2}{|l|}{ Placing rat on the floor (normal $=0$; maximum $=3$ ) } \\
\hline Normal walk & 0 \\
\hline Inability to walk straight & 1 \\
\hline Circling toward the paretic side & 2 \\
\hline Fall down to the paretic side & 3 \\
\hline \multicolumn{2}{|l|}{ Sensory tests } \\
\hline Placing test (visual and tactile test) & $\mathrm{I}$ \\
\hline $\begin{array}{l}\text { Proprioceptive test (deep sensation, pushing the paw } \\
\text { against the table edge to stimulate limb muscles) }\end{array}$ & 2 \\
\hline \multicolumn{2}{|l|}{ Beam balance tests $($ normal $=0$; maximum $=6$ ) } \\
\hline Balances with steady posture & 0 \\
\hline Grasps side of beam & 1 \\
\hline Hugs the beam and one limb falls down from the beam & 2 \\
\hline $\begin{array}{l}\text { Hugs the beam and two limbs fall down from the } \\
\text { beam or spins on beam }(60 \mathrm{~s})\end{array}$ & 3 \\
\hline Attempts to balance on the beam but falls off ( $40 \mathrm{~s})$ & 4 \\
\hline Attempts to balance on the beam but falls off $(20 \mathrm{~s})$ & 5 \\
\hline $\begin{array}{l}\text { Falls off: no attempt to balance or hang on to the } \\
\text { beam }(20 \mathrm{~s})\end{array}$ & 6 \\
\hline \multicolumn{2}{|l|}{ Reflexes absent and abnormal movements } \\
\hline $\begin{array}{l}\text { Pinna reflex (head shakes when touching the } \\
\text { auditory meatus) }\end{array}$ & 1 \\
\hline $\begin{array}{l}\text { Corneal reflex (eye blinks when lightly touching the } \\
\text { cornea with cotton) }\end{array}$ & 1 \\
\hline $\begin{array}{l}\text { Startle reflex (motor response to a brief noise from } \\
\text { snapping a clipboard paper) }\end{array}$ & 1 \\
\hline Seizures, myoclonus and myodystony & 1 \\
\hline Maximum points & 18 \\
\hline
\end{tabular}


injury, 7-12 points indicated moderate injury and $1-6$ points indicated mild injury.

\section{Grip strength test}

The grip strength test was measured in the left and right forelimbs of each rat using a grip strength meter (RWD Life Science and Technology Co., Ltd., Shenzhen, China). It was measured at 1, 4, 7 and 14 days after MCAO. Each rat was fixed horizontally and then pulled steadily by the root of the tail away from the bar until its grip was broken. The peak of the grip strength was measured. Each rat was subjected to five such trials, and the average value was used as the grip strength.

\section{Morris water maze test}

The Morris water maze test was carried out at 1, 4, 7 and 14 days after MCAO. Before test, all animals were trained four times each day for 6 days before surgery. Briefly, rats were slightly placed into the water from the direction of the face to the wall of the pool. The time to find the hidden platform under water at $1 \mathrm{~cm}$ was recorded and determined as escape latency. Animals were allowed to swim for $60 \mathrm{~s}$ to find the hidden platform at each trial. If the rat could not find it after $60 \mathrm{~s}$, the escape latency was considered as $60 \mathrm{~s}$. The average escape latency time of last four tests was regarded as the baseline.

\section{Infarct volume assessment}

The volume of infarct area was assessed at 1, 4, 7 and 14 days after the start of reperfusion. For this assessment, the brains were rapidly isolated and sliced into 2-mm thick coronal sections. The sections were stained with 2,3,5-triphenyltetrazolium chloride (TTC) at $37^{\circ} \mathrm{C}$ for $10 \mathrm{~min}$ and then fixed with $4 \%$ paraformaldehyde. As TTC was converted into a red dye when taken up into normal cells, the damage regions appeared as completely white areas. The stained sections were photographed and analyzed using the AutoCAD analysis software (San Rafael, CA, USA).

\section{Histopathology}

Rats were sacrificed $24 \mathrm{~h}$ after the start of reperfusion. Brain cortices were departed rapidly and then postfixed for $24 \mathrm{~h}$ in the $4 \%$ paraformaldehyde. The tissues were dehydrated in graded ethanol, cleared in xylene, embedded in paraffin wax and cut into continuous coronary slices. The sections were then stained with hematoxylin-eosin (HE) for histopathological observation.

\section{Oxidative stress}

Cortex tissues from each group were collected at 14 days after reperfusion. Each tissue was placed into the cold radio immunoprecipitation assay (RIPA) lysis buffer (Beyotime Institute of Biotechnology, Shanghai, China) for dissociation with a polytron homogenizer, followed by centrifugation at $3,000 \mathrm{rpm} / \mathrm{min}$ for $15 \mathrm{~min}$. The supernatant was collected for superoxide dismutase (SOD), malonaldehyde (MDA), catalase (CAT) and glutathione peroxidase (GSH-PX) measurements using the total SOD assay kit with WST-8 (Beyotime Institute of Biotechnology), lipid peroxidation MDA assay kit (Beyotime Institute of Biotechnology), CAT assay kit (Beyotime Institute of Biotechnology) and total GSH-PX assay kit (Beyotime Institute of Biotechnology), respectively. The assay was conducted according to the manufacturer's instructions. The absorbance of each final solution was measured at $450 \mathrm{~nm}$ (SOD), $532 \mathrm{~nm}$ (MDA), $520 \mathrm{~nm}$ (CAT) and $340 \mathrm{~nm}$ (GSH-PX). The SOD activity was calculated according to the SOD standard control and expressed as U/mg protein. The MDA content was expressed as $\mu \mathrm{mol} / \mathrm{mg}$ protein. CAT activity and GSH-Px activity were expressed as U/mg protein.

\section{Western blot}

The total proteins were extracted from the ischemic cortex. Western blot assays were performed according to the manufacturer's procedures. Equal samples from each group were loaded onto and separated by sodium dodecyl sulfate polyacrylamide gel electrophoresis and transferred onto polyvinylidene fluoride membranes. The membranes were blocked in 5\% bovine serum albumin for $1 \mathrm{~h}$ at room temperature and then incubated with primary antibody (c-caspase-3, c-caspase-9, Cyt-C, Bcl-2, Bax, $\beta$-actin [1:1,000; Santa Cruz Biotechnology Inc., Dallas, TX, USA]) overnight at $4^{\circ} \mathrm{C}$, followed by secondary antibody conjugated to horseradish peroxidase for $2 \mathrm{~h}$. Immunoblot was visualized with enhanced chemiluminescence Western blot detection reagents and analyzed using the Image-Pro Plus V7.0 software (Rockville, MD, USA).

\section{Statistical analysis}

All data were analyzed by one-way analysis of variance (ANOVA) using the Statistical Package for the Social Sciences (SPSS) 16.0 software (SPSS Inc., Chicago, IL, USA). Multiple comparison post hoc tests between groups were performed using the least-significant difference test. Data were presented as mean \pm standard error (SE), and differences between groups were considered significant at $P<0.05$. 


\section{Results}

\section{Curdione reduced infarct size and} neurological deficits and promoted motor function and cognitive function recovery in MCAO rats

We first investigated the neuroprotective effect of curdione in rat focal cerebral ischemia. The infarct size of animals was determined by TTC staining. After $24 \mathrm{~h}$ of reperfusion, there was no cerebral injury in the sham group. Whereas the infarct area of the MCAO group was obviously observed, and curdione significantly decreased infarct volumes from $28.4 \% \pm 3.01 \%$ to $11.5 \% \pm 1.12 \%$. Similar data were obtained at 4, 7 and 14 days after MCAO, as represented in Figure 2.

The neuroprotective effects of curdione were also assessed based on behavioral tests, including the NSS test and Morris water maze task. All experiments were tested at 1, 4, 7 and 14 days after MCAO. Figure $3 \mathrm{~A}$ exhibits that the MCAO group rats showed the highest neurological deficit score and curdione treatment significantly decreased the score.

In the grip strength test, the grip behavior of the rats in the model group was worse than that of the rats in the sham group. However, the grip strength of the rats in the curdione treatment group was considerably recovered (Figure 3B).

A
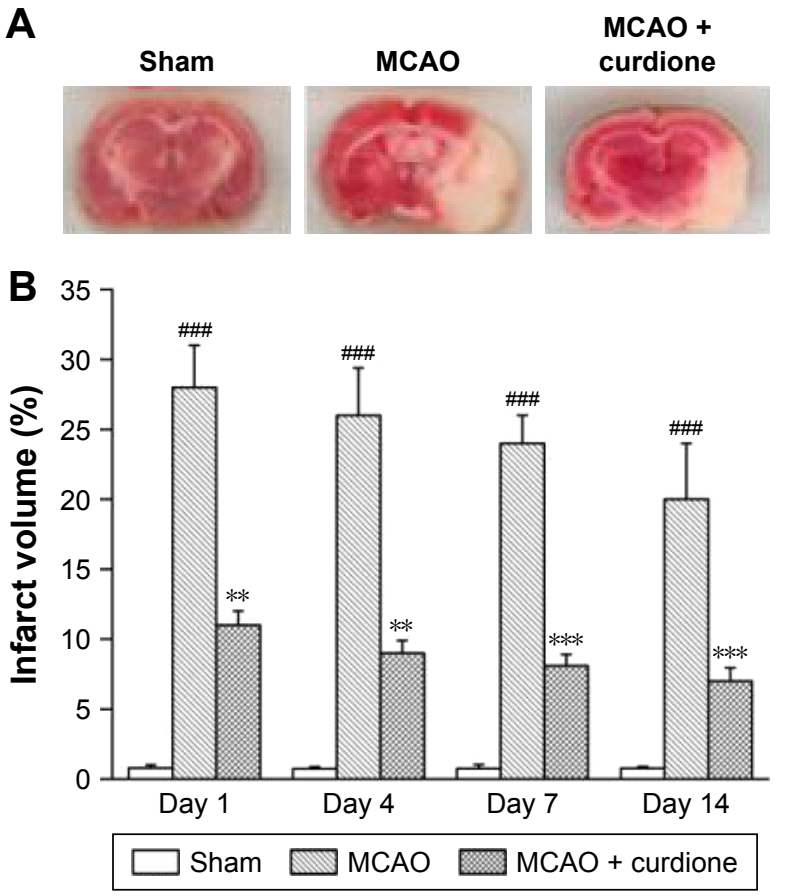

Figure 2 Curdione reduced cerebral infarction volume in MCAO rats. Notes: (A) Representative infarct volume is shown on TTC staining after reperfusion. (B) Quantitative evaluation of infract volume ratio in each group. All data were presented as mean $\pm S E$. $P<0.00$ I vs sham and $* * P<0.0$ I, $* * * P<0.00$ I vs MCAO. $n=10$.

Abbreviations: MCAO, middle cerebral artery occlusion; TTC, 2,3,5triphenyltetrazolium chloride; SE, standard error.
The Morris water maze task showed that MCAO rats spent more time to look for the hidden platform compared to the sham group. However, the curdione treatment rats attained a significantly lower time to search the platform compared to $\mathrm{MCAO}$ rats (Figure 3C).

\section{Curdione recovered neuronal morphologic damages in MCAO rats}

To further identify the neuroprotective effect of curdione, we evaluated the histopathological changes in cerebral cortices after $24 \mathrm{~h}$ reperfusion following $2 \mathrm{~h} \mathrm{MCAO}$ with HE staining. As shown in Figure 4, the neurons in the sham group were tightly aligned with distinct nucleoli and moderate size. Those in the MCAO group exhibited obvious pathological abnormalities with loosed arranged neurons, pyknotic nucleus and loss or dark color staining. By contrast, these histopathological alterations were reversed by curdione treatment.

\section{Curdione defended against oxidative damage in MCAO rats}

To demonstrate whether the anti-oxidative property was involved in the neuroprotective effect of curdione, we detected the activities of endogenous antioxidant enzymes in ischemic cortex at 14 days after MCAO. Results showed that MCAO injury decreased the activities of SOD, CAT and GSH-PX significantly compared to the sham group. However, curdione treatment significantly increased the activities of SOD, CAT and GSH-PX compared with the MCAO group, making that close to the normal level. At the same time, we also detected the MDA level in each group, a marker of lipid peroxidation. The MDA level of MCAO rats was almost twice than that in the sham group, while curdione suppressed the increase in the MDA content caused by the injury with cerebral ischemia/reperfusion (Table 2).

\section{Curdione regulated the expression of Bcl-2, Bax, c-caspase-3, c-caspase- 9 and Cyt-C proteins in ischemic cortex of MCAO rats}

To further explore the underlying mechanisms of curdione, we assayed apoptosis-related proteins in the ischemic cortex by Western blot analysis. Figure 5 illustrates that MCAO injury upregulated Bax expression 2.08 times and downregulated Bcl-2 expression 0.28 times, thereby decreasing the Bcl-2/Bax ratio compared with the sham group, but curdione recovered the changes significantly. In addition, Cyt-C protein levels were 3.19 times higher in the MCAO group compared with those in the sham group, and the expression of c-caspase- 3 and 


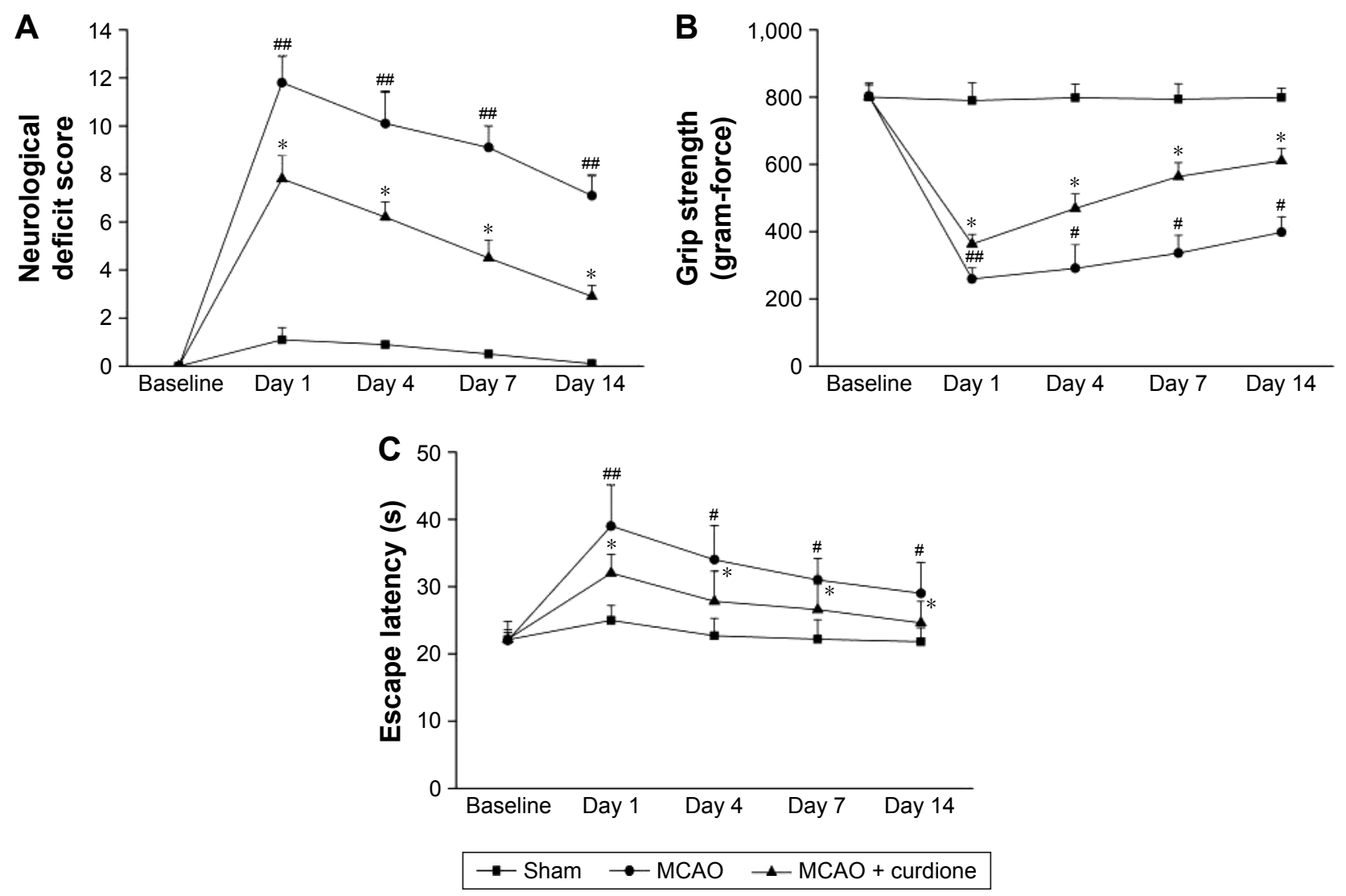

Figure 3 Curdione promoted neurological function recovery in MCAO rats.

Notes: (A) NSS test scores, (B) the grip strength of each group and $(\mathbf{C})$ escape latency of each group in the Morris water maze test. Data were expressed as mean \pm SE. ${ }^{\#} P<0.05,{ }^{\#} P<0.01$ vs sham and $* P<0.05$ vs MCAO. $n=10$.

Abbreviations: MCAO, middle cerebral artery occlusion; NSS, neurological severity score; SE, standard error.

c-caspase-9 were also elevated significantly in 14 days after MCAO reperfusion injury. Treatment with curdione reversed the effect of MCAO on Cyt-C, c-caspase-3 and c-caspase-9 induction, suggesting that curdione possessed anti-apoptotic property within the context of MCAO reperfusion injury.

\section{Discussion}

Developing safe and effective compounds from natural products to treatment for different ailments has become the focus of researchers. ${ }^{5}$ Lack of therapeutic drug for stroke has raised the attention to test possible beneficial effects of small compounds from herbal origin. Curdione is one of the most active ingredients in E-zhu. Even though curdione has been validated to possess diverse pharmacological activities, its neuroprotective potential is largely unexplored.

The present study evaluated the potential protective effect of curdione on the cerebral ischemia/reperfusion-induced brain injury in experimental animals. We found that curdione significantly decreased the neurological deficit scores and infarct size in rats with $2 \mathrm{~h}$ of ischemia followed by $22 \mathrm{~h}$ of
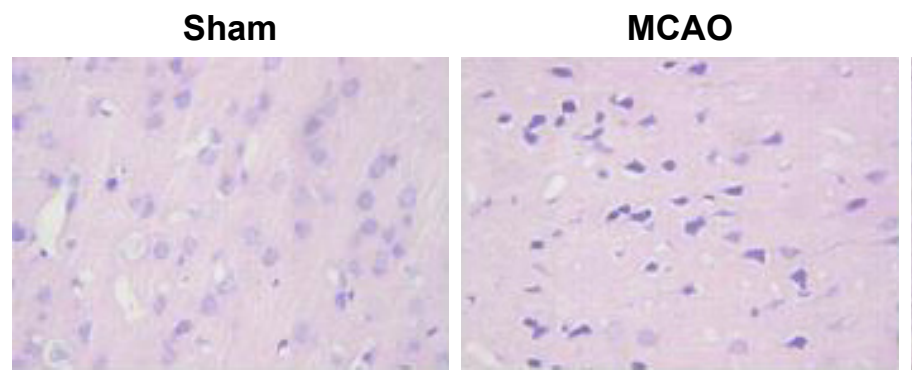

\section{MCAO + curdione}

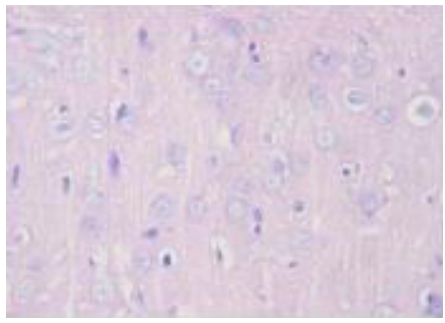

Figure 4 Curdione recovered neuronal morphologic damages after cerebral ischemia reperfusion in MCAO rats.

Notes: The histopathological changes in cerebral cortices were evaluated after $24 \mathrm{~h}$ reperfusion following $2 \mathrm{~h}$ MCAO with HE staining. Magnification $\times 200$.

Abbreviations: MCAO, middle cerebral artery occlusion; HE, hematoxylin-eosin. 
Table 2 Curdione defended against oxidative damage in MCAO rats

\begin{tabular}{llllll}
\hline Groups & $\mathbf{n}$ & $\begin{array}{l}\text { MDA }(\mu \mathrm{mol} / \mathrm{mg} \\
\text { protein) }\end{array}$ & $\begin{array}{l}\text { CAT (U/mg } \\
\text { protein) }\end{array}$ & $\begin{array}{l}\text { GSH-PX (U/mg } \\
\text { protein) }\end{array}$ & $\begin{array}{l}\text { SOD (U/mg } \\
\text { protein) }\end{array}$ \\
\hline Sham & 10 & $4.6 \pm 0.84$ & $20.2 \pm 2.34$ & $18.1 \pm 3.42$ & $13.9 \pm 3.56$ \\
MCAO & 10 & $9.5 \pm 1.86^{\# \#}$ & $11.6 \pm 1.63^{\#}$ & $11.3 \pm 1.32^{\#}$ & $9.1 \pm 1.13^{\#}$ \\
MCAO + curdione & 10 & $7.2 \pm 1.02^{*}$ & $15.6 \pm 2.64^{*}$ & $14.2 \pm 3.23^{*}$ & $11.5 \pm 1.83^{*}$ \\
\hline
\end{tabular}

Notes: All data were presented as mean \pm SE. ${ }^{\#} P<0.05,{ }^{\# P}<0.01$ vs sham and $* P<0.05$ vs MCAO. $n=10$.

Abbreviations: MCAO, middle cerebral artery occlusion; MDA, malonaldehyde; CAT, catalase; GSH-PX, glutathione peroxidase; SOD, superoxide dismutase; $\mathrm{SE}$, standard error.

reperfusion. In addition, curdione also alleviated the cerebral histological damage and accelerated the improvement of motor and cognitive functions. Furthermore, curdione markedly brought down the levels of MDA, as well as antagonized the decreases in SOD, CAT and GSH-PX activities induced by cerebral ischemia/reperfusion.

Oxidative stress has been reported to be the main pathogenesis in the progression of ischemia/reperfusion. ${ }^{17}$
A
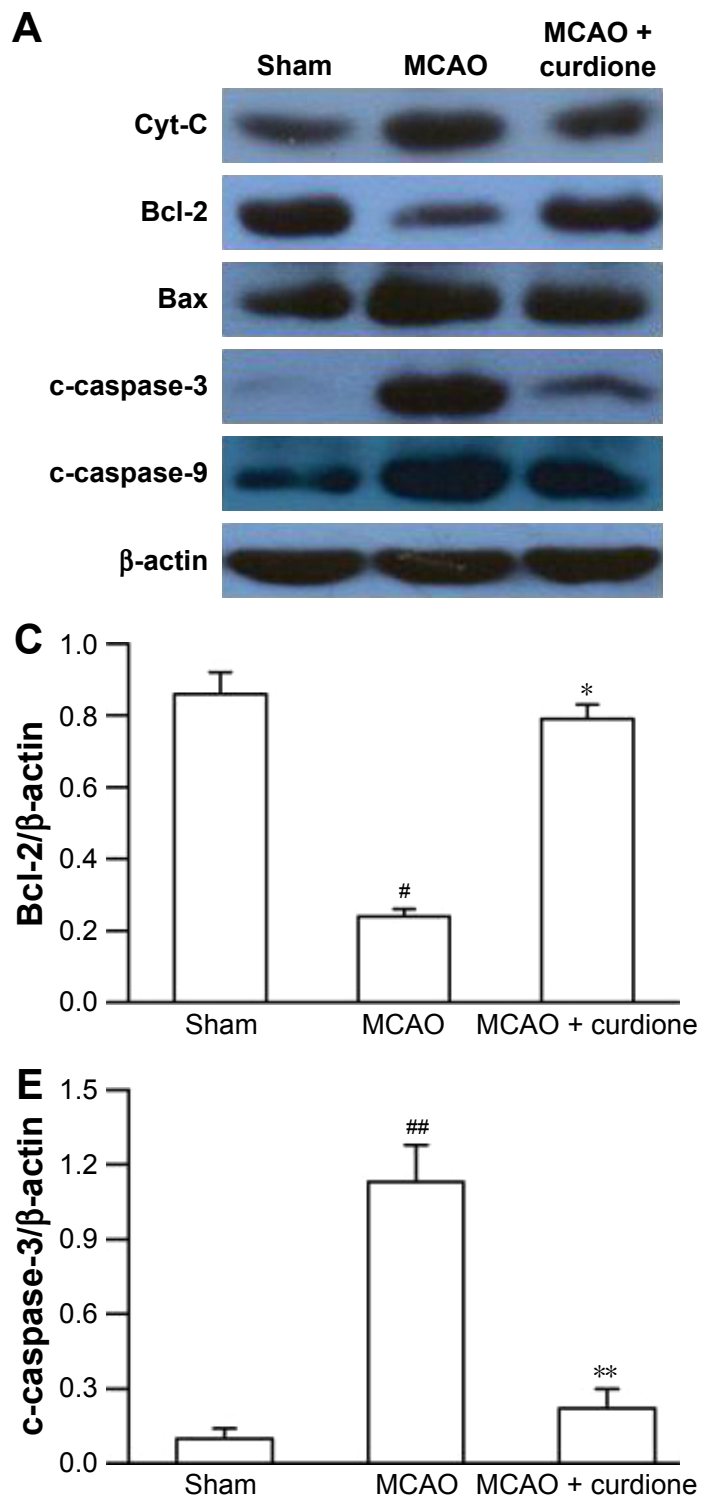

B
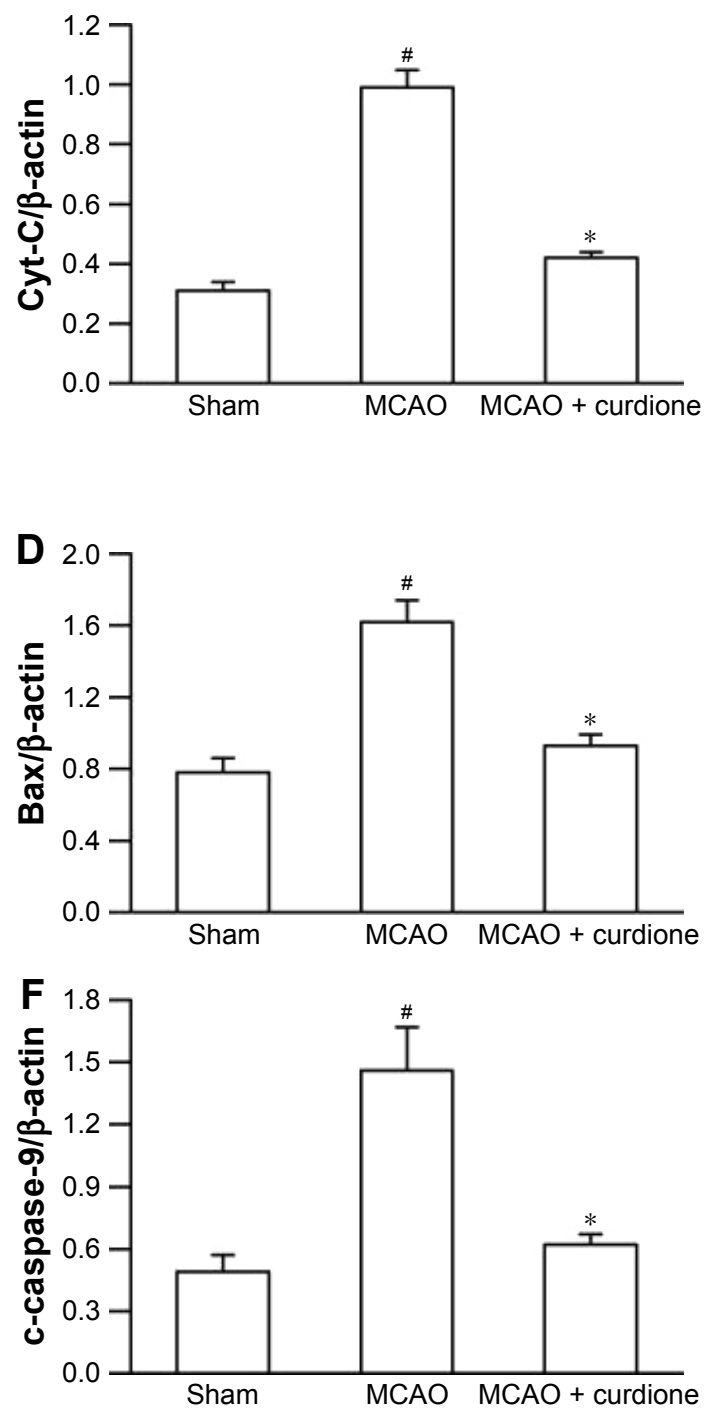

Figure 5 Curdione inhibited apoptosis induced by MCAO in ischemic cortex.

Notes: (A) Representative immunoblots of Cyt-C, Bcl-2, Bax, c-caspase-3, c-caspase-9 and $\beta$-actin. Quantification of protein levels in experimental groups: (B) Cyt-C, (C) Bcl-2, (D) Bax, (E) c-caspase-3 and (F) c-caspase-9. The intensity of each band was normalized to $\beta$-actin. All data were presented as mean \pm SE. ${ }^{\prime}<<0.05$, $P<0.0 \mathrm{I}$ vs Sham, $* \mathrm{p}<0.05$ and $* * \mathrm{p}<0.0$ I vs MCAO. $\mathrm{n}=3$.

Abbreviations: MCAO, middle cerebral artery occlusion; SE, standard error. 
Excess reactive oxygen species (ROS) could induce severe lipid damage, which creates vast cytotoxic MDA via lipid peroxidation..$^{18}$ While curdione blocked the increase in the MDA content caused by the injury with cerebral ischemia/ reperfusion. Under normal circumstances, there is a balance between ROS generation and the endogenous antioxidant systems in the cell. However, ischemia and reperfusion disturb the balance of the endogenous antioxidant system, which increases oxidative stress by the overproduction of cellular ROS and ultimately leads to neuronal death. ${ }^{19-21}$ SOD, CAT and GSH-PX, as the endogenous antioxidant enzymes, are believed to play vital roles in the defense mechanisms against free radical-induced oxidative stress. ${ }^{22,23} \mathrm{SOD}$ is the first defendant line to confront oxidative stress by catalyzing the dismutation of superoxide anion to hydrogen peroxide and preventing the formation of the hydroxyl radical. CAT and GSH-PX are the second line of defense by transferring ROS into water and molecular oxygen. ${ }^{24} \mathrm{All}$ of these endogenous antioxidants were decreased during the ischemia/reperfusion, whereas curdione treatment reversed the effects. Our results indicated that the neuroprotective potential of curdione may be via modulation of these endogenous antioxidants.

During intracellular ROS overproduction, the loss of outer mitochondrial membrane integrity leads to amounts of mitochondrial protein, including Cyt-C release from mitochondrial matrix to cytoplasm. Cyt-C release along with complete loss of membrane potential activates the caspase cascades, ultimately causing apoptosis..$^{25}$ Caspases transduce and execute apoptotic signaling. ${ }^{26} \mathrm{Bcl}-2$ regulates the mitochondrial permeability transition pore opening in opposition to Bax, blocking Cyt-C release, inhibiting caspase activity and decreasing cell apoptosis. ${ }^{27}$ Modulating the ratio of $\mathrm{Bcl}-2 / \mathrm{Bax}$ may be important to apoptosis. ${ }^{28}$ Our results showed that curdione significantly increased the Bcl-2/Bax ratio and decreased the expression of Cyt-C, c-caspase-3 and c-caspase- 9 in ischemic brains, indicating an anti-apoptotic effect of curdione on cerebral ischemia reperfusion injury.

\section{Conclusion}

In summary, the present study first demonstrated that the neuroprotective effect of curdione on cerebral ischemia/ reperfusion induced brain injury and the effect partially due to the anti-oxidative and anti-apoptotic ability of curdione. However, our conclusions were all based on the animal testing alone, and other potential involving signaling pathways should be illustrated on the following research. The present study showed a high possibility of curdione to become a candidate drug for the cerebral stroke therapy.

\section{Acknowledgment}

This study was supported by the Science and Technology Support Program of Gansu Province, China (Grant No 1504FKCA086), and Natural Science Foundation of Gansu Province, China (Grant No 1606RJZA116).

\section{Disclosure}

The authors report no conflicts of interest in this work.

\section{References}

1. Feigin VL. Stroke epidemiology in the developing world. Lancet. 2005;365(9478):2160-2161.

2. Flynn RW, MacWalter RS, Doney AS. The cost of cerebral ischaemia. Neuropharmacology. 2008;55(3):250-256.

3. Molina CA, Alvarez-Sabín J. Recanalization and reperfusion therapies for acute ischemic stroke. Cerebrovasc Dis. 2009;27(suppl 1): 162-167.

4. Goldstein LB, Rothwell PM. Advances in prevention and health services delivery. Stroke. 2008;39(2):258-260.

5. Yu XQ, Xue CC, Zhou ZW, et al. In vitro and in vivo neuroprotective effect and mechanisms of glabridin, a major active isoflavan from Glycyrrhiza glabra (licorice). Life Sci. 2008;82(1-2):68-78.

6. China Medical Science and Technology Press. Pharmacopoeia of the People's Republic of China. Subdivision of 2010 Edition. Beijing: China Medical Science and Technology Press; 2010: 257-258.

7. Lai YC, Chyau CC, Mau JL, et al. Antimicrobial activity and cytotoxicity of the essential oil of Curcuma zedoaria. Am J Chin Med. 2004; 32(2):281-290.

8. Makabe H, Maru N, Kuwabara A, Kamo T, Hirota M. Anti-inflammatory sesquiterpenes from Curcuma zedoaria. Nat Prod Res. 2006;20(7): 680-685.

9. Li J, Bian WH, Wan J, et al. Curdione inhibits proliferation of MCF-7 cells by inducing apoptosis. Asian Pac J Cancer Prev. 2014;15(22): 9997-10001.

10. Xia Q, Wang X, Xu DJ, Chen XH, Chen FH. Inhibition of platelet aggregation by curdione from Curcuma wenyujin essential oil. Thromb Res. 2012;130(3):409-414.

11. Zhang JS, Guan J, Yang FQ, Liu HG, Cheng XJ, Li SP. Qualitative and quantitative analysis of four species of Curcuma rhizomes using twice development thin layer chromatography. J Pharm Biomed Anal. 2008;48(3):1024-1028.

12. Hikino H, Sakurai Y, Takahashi H, Takemoto T. Structure of curdione. Chem Pharm Bull. 1967;15(9):1390-1394.

13. Dohare P, Garg P, Sharma U, Jagannathan NR, Ray M. Neuroprotective efficacy and therapeutic window of curcuma oil: in rat embolic stroke model. BMC Complement Altern Med. 2008;8:55-74.

14. Longa EZ, Weinstein PR, Carlson S, Cummins R. Reversible middle cerebral artery occlusion without craniectomy in rats. Stroke. 1989; 20(1):84-91.

15. Chen J, Li Y, Wang L, et al. Therapeutic benefit of intravenous administration of bone marrow stromal cells after cerebral ischemia in rats. Stroke. 2001;32(4):1005-1011.

16. Germano AF, Dixon CE, d'Avella D, Hayes RL, Tomasello F. Behavioral deficits following experimental subarachnoid hemorrhage in the rat. J Neurotrauma. 1994;11(3):345-353.

17. Reynolds A, Laurie C, Mosley RL, Gendelman HE. Oxidative stress and the pathogenesis of neurodegenerative disorders. Int Rev Neurobiol. 2007;82:297-325.

18. Schettler V, Methe H, Staschinsky D, Schuff-Werne P, Müller GA, Wieland E. Review: the oxidant/antioxidant balance during regular low density lipoprotein apheresis. Ther Apher. 1999;3(3):219-226.

19. Ahmed E, Donovan T, Yujiao L, Zhang Q. Mitochondrial targeted antioxidant in cerebral ischemia. J Neurol Neurosci. 2015;6(2):17. 
20. Tabassum R, Vaibhav K, Shrivastava P, et al. Perillyl alcohol improves functional and histological outcomes against ischemia-reperfusion injury by attenuation of oxidative stress and repression of COX-2, NOS-2 and NF- $\kappa \mathrm{B}$ in middle cerebral artery occlusion rats. Eur $J$ Pharmacol. 2015;747:190-199.

21. Floyd RA. Role of oxygen free radicals in carcinogenesis and brain ischemia. FASEB J. 1990;4(9):2587-2597.

22. Ahmad S, Yousuf S, Ishrat T, et al. Effect of dietary sesame oil as antioxidant on brain hippocampus of rat in focal cerebral ischemia. Life Sci. 2006;79(20):1921-1928.

23. Zhang Y, Milatovic D, Aschner M, Feustel PJ, Kimelberg HK. Neuroprotection by tamoxifen in focal cerebral ischemia is not mediated by an agonist action at estrogen receptors but is associated with antioxidant activity. Exp Neurol. 2007;204(2):819-827.

24. Djordjevic A, Spasic S, Jovanovic-Galovic A, Djordjevic R, GruborLajsic G. Oxidative stress in diabetic pregnancy: SOD, CAT and GSH-Px activity and lipid peroxidation products. J Matern Fetal Neonatal Med. 2004;16(6):367-372.
25. Wang Y, Li X, Wang X, et al. Ginsenoside Rd attenuates myocardial ischemia/reperfusion injury via Akt/GSK-3 $\beta$ signaling and inhibition of the mitochondria-dependent apoptotic pathway. PLoS One. 2013;8(8):e70956.

26. Yao K, Ye P, Zhang L, Tan J, Tang X, Zhang Y. Epigallocatechin gallate protects against oxidative stress-induced mitochondria-dependent apoptosis in human lens epithelial cells. Mol Vis. 2008;14:217-223.

27. Cook SA, Sugden PH, Clerk A. Regulation of bcl-2 family proteins during development and in response to oxidative stress in cardiac myocytes: association with changes in mitochondrial membrane potential. Circ Res. 1999;85(10):940-949.

28. Tamm I, Schriever F, Dorken B. Apoptosis: implications of basic research for clinical oncology. Lancet Oncol. 2001;2(1):33-42.

\section{Publish your work in this journal}

Neuropsychiatric Disease and Treatment is an international, peerreviewed journal of clinical therapeutics and pharmacology focusing on concise rapid reporting of clinical or pre-clinical studies on a range of neuropsychiatric and neurological disorders. This journal is indexed on PubMed Central, the 'PsycINFO' database and CAS, and is the official journal of The International Neuropsychiatric Association (INA). The manuscript management system is completely online and includes a very quick and fair peer-review system, which is all easy to use. Visit http://www.dovepress.com/testimonials.php to read real quotes from published authors. 